\title{
Lateral Size Dependence in FRET between Semiconductor Nanoplatelets and Conjugated Fluorophores
}

\author{
Henry Halim, David Trieb, Niklas Huber, María Martínez-Negro, Lars-Arne Meyer, Thomas Basché, \\ Svenja Morsbach, Kai A. I. Zhang, and Andreas Riedinger*
}

Cite This: J. Phys. Chem. C 2020, 124, 25028-25037

Read Online

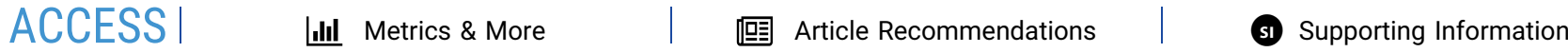

ABSTRACT: Sensitization of organic molecules by semiconductor nanocrystals is a promising way to boost the absorption of the former, important for applications in fluorescence labeling and photocatalysis. Semiconductor nanoplatelets provide the opportunity of increasing the absorption cross section by increasing the lateral dimension without inducing changes to the band gap. However, the lateral size dependence of FRET in nanoplatelet small-molecule conjugates is unknown. Our FRET study supports the view that

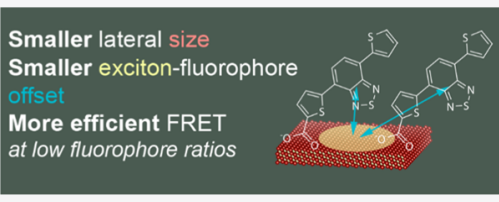
excitons in nanoplatelets are localized in a small area and spatially distributed in a stochastic fashion. In larger nanoplatelets, the likelihood of an offset between the position of the exciton and the acceptor increases. Although this could be mitigated by increasing the number of fluorophores, excessive coverage of the nanoplatelets with the fluorophores leads to severe self-quenching. Based on these results, we predict that using few fluorophores on nanoplatelets with edge lengths of $\sim 9$ nm would optimize the delicate balance between maximizing the absorption cross section of the nanoplatelets and minimizing the likelihood of exciton-fluorophore offset and self-quenching simultaneously, thus leading to optimal FRET conditions.

\section{INTRODUCTION}

Organic fluorophores play an indispensable role in a variety of applications, including bioimaging, sensing, and photocatalysis. $^{1-5}$ Their versatility arises from the fact that their properties can be fine-tuned by changing their molecular structures. However, their absorption cross sections are lower and of narrow bandwidth compared to those of inorganic materials. ${ }^{6}$ This limits the choice of excitation wavelengths and requires higher excitation powers for image acquisition with organic fluorophores in (bio) imaging applications and increased loading of the photocatalyst and/or high excitation powers in photocatalysis with organic fluorophores. Semiconductor nanocrystals possess size-tunable absorption bands and absorption cross sections, ${ }^{7-9}$ are relatively photostable, ${ }^{10}$ and can act as a sensitizer for molecules in their proximity. ${ }^{11-18}$ Therefore, the combination of semiconductor nanocrystals with organic fluorophores could be useful for applications where excitation intensities are low, e.g., absorption of sunlight without concentrators. To make this principle work, the energy absorbed by the nanocrystals must be transferred to the organic fluorophore, e.g., via FRET. ${ }^{19}$

FRET occurs when the nanocrystal and the organic fluorophore are in close proximity, oriented at a suitable angle, and if there is sufficient spectral overlap between the nanocrystal emission and the fluorophore absorption band. Since the absorption and emission bands of quasi-spherical nanocrystals (quantum dots, QDs) are size-dependent, concurrent and independent tuning of the absorption cross section (by the size) and the spectral overlap (also by size) is problematic. Anisotropic semiconductor nanoplatelets
(NPLs), ${ }^{20}$ also known as colloidal quantum wells, overcome this issue. The atomically precise thickness of a few atomic layers of NPLs (e.g., made from CdSe) is smaller and the lateral extension larger than the Bohr exciton radius. ${ }^{21}$ Due to this highly anisotropic structure, NPLs exhibit large absorption cross sections and the spectral position of the absorption and emission bands only depend on the thickness, while the lateral extensions determine the absorption cross section. ${ }^{8}$ Furthermore, their flat and well-defined surfaces should simplify their surface chemistry.

This implies that it should be beneficial to conjugate NPLs with organic fluorophores in a donor-acceptor FRET couple. This arrangement would allow us to significantly broaden the absorption bandwidth and boost the absorption cross section of the conjugate using laterally large NPLs as the sensitizer.

However, recently Brumberg et al. measured the size of the exciton in NPLs using magneto-optical spectroscopy and concluded that excitons are not delocalized across the lateral dimensions of the NPL but rather localized within a radius in the range of $1.0-1.5 \mathrm{~nm}^{22}$ By employing transient absorption spectroscopy, Li et al. $^{23}$ and Morgan et al. ${ }^{24}$ have reported much larger exciton sizes in the range of several tens of nanometers. While the discrepancies of the various measure-

Received: July 7, 2020

Revised: October 19, 2020

Published: November 2, 2020 
ments are still under debate, a common finding is exciton localization at room temperature. Thus, in laterally extended NPLs, the fluorophore would have to be in close proximity to the localized exciton to allow for efficient energy transfer. For FRET, this would mean a distance not much further than the Förster radius. Based on this, it would be important for practical applications to know the largest possible NPL size one can use as a sensitizer, without losing too much of the FRET efficiency due to large exciton-acceptor separation distance.

Here, we present our findings on FRET between 3 monolayer (ML, defined as the number of Se layers in zincblende CdSe across the thickness) thick CdSe NPLs and a carboxyl-functionalized diethienyl benzothiadiazole derivative [DBTCA, 5-(7-(thiophen-2-yl)benzo[c] [1,2,5] thiadiazol-4-yl)thiophene-2-carboxylic acid]. DBTCA has an excellent spectral overlap with $3 \mathrm{ML}$ CdSe NPLs' photoluminescence (PL), and its derivatives have found applications in the field of photovoltaics $^{25,26}$ and photocatalysis. ${ }^{27,28}$ Therefore, we used it as a model system for our study, using the carboxyl group of DBTCA as an anchor for the Cd-terminated NPL facets. By varying the DBTCA coverage on the surface of differently sized NPLs, we found a relationship between the size and the FRET efficiency. The analysis of the FRET behaviors observed in this work confirms that excitons are indeed of a small radius and stochastically distributed in CdSe NPLs. This shows that an optimum lateral size and fluorophore coverage exists and simply increasing the absorption cross section using larger NPLs is detrimental to the sensitization efficiency of small organic molecules. This finding serves as a useful guideline for developing more efficient nanocrystal sensitizer systems for FRET-based photocatalysis and fluorescent imaging.

\section{EXPERIMENTAL SECTION}

Chemicals. $n$-Hexane (95\%) was purchased from Fischer Scientific. Methyl acetate (99\%) was purchased from Merck KGaA. 1-Octadecene (ODE, 90\%) and cadmium acetate dihydrate (98\%) were purchased from Acros Organics. Selenium powder (99.999\%) and methanol (100\%) were purchased from Alfa-Aesar. Toluene (99.8\%) was purchased from VWR Chemicals. Oleic acid (90\%), cadmium nitrate tetrahydrate (98\%), and myristic acid (>98\%) were purchased from Aldrich. All chemicals were used as received without further purification.

Synthesis of Cadmium Myristate. Cadmium myristate was prepared from its nitrate salt using standard literature methods. $^{29-31}$ Cadmium nitrate $(1.23 \mathrm{~g})$ was dissolved in 10 $\mathrm{mL}$ of methanol. Sodium myristate $(3.13 \mathrm{~g})$ was dissolved in $100 \mathrm{~mL}$ of methanol. The solutions were stirred well separately for $10 \mathrm{~min}$ until they were clear. Then, they were mixed together and stirred for $30 \mathrm{~min}$ at room temperature. The resulting white precipitate ( $\mathrm{Cd}$ myristate) was filtered, rinsed three times using cold methanol, and dried under vacuum overnight. The white powder was stored at room temperature in the dark.

Synthesis of Bis(stearoyl)selenide. Bis(stearoyl)selenide was prepared using $\mathrm{LiAlHSeH}$ as the selenating agent. ${ }^{32}$ To prepare $\mathrm{LiAlHSeH} \mathrm{LiAlH}_{4}(0.76 \mathrm{~g}, 20 \mathrm{mmol})$ was added to a suspension of Se powder $(1.92 \mathrm{~g}, 24 \mathrm{mmol})$ in $200 \mathrm{~mL}$ of THF at $-10{ }^{\circ} \mathrm{C}$ under an argon atmosphere. The mixture was stirred for $30 \mathrm{~min}$, and $\mathrm{LiAlHSeH}$ was formed in situ as a grayish dispersion. Then, $80 \mathrm{mmol}(27.2 \mathrm{~mL})$ of stearoyl chloride was slowly added to the dispersion of $20 \mathrm{mmol}$ of LiAlHSeH. The mixture was stirred at $-10{ }^{\circ} \mathrm{C}$ under a nitrogen atmosphere. After $2 \mathrm{~h}, 5 \mathrm{~mL}$ of deionized water was added to quench unreacted reagents. The organic reaction mixture was diluted with $300 \mathrm{~mL}$ of diethyl ether and washed four times with 100 $\mathrm{mL}$ of brine. Bis(stearoyl)selenide was crystallized at $20^{\circ} \mathrm{C}(12$ $\mathrm{h}$ ), filtered, and dried under high vacuum for $12 \mathrm{~h}$. The resulting white flakes were stored in a nitrogen-filled glovebox.

Synthesis of Laterally Small 3 ML NPLs (NPL 150). ${ }^{33}$ In a three-necked flask, $127.5 \mathrm{mg}(0.225 \mathrm{mmol})$ of cadmium myristate and $17.3 \mathrm{mg}(0.065 \mathrm{mmol})$ of $\mathrm{Cd}(\mathrm{OAc})_{2} \cdot 2 \mathrm{H}_{2} \mathrm{O}$ were dispersed in $15 \mathrm{~mL}$ of ODE. This mixture was heated to 100 ${ }^{\circ} \mathrm{C}$ and degassed under vacuum for $15 \mathrm{~min}$. Afterward, the mixture was heated to $140{ }^{\circ} \mathrm{C}$ under argon. At this temperature, a solution of $45.8 \mathrm{mg}(0.075 \mathrm{mmol})$ of bis(stearoyl)selenide dissolved in $1 \mathrm{~mL}$ of anhydrous toluene was added with a syringe. One minute later, $52 \mathrm{mg}(0.195$ $\mathrm{mmol})$ of $\mathrm{Cd}(\mathrm{OAc})_{2} \cdot 2 \mathrm{H}_{2} \mathrm{O}$ was added. The mixture was kept heated at $140{ }^{\circ} \mathrm{C}$ with an oil bath for 5 days. After 5 days, the mixture was cooled to room temperature. Then, $0.5 \mathrm{~mL}$ of oleic acid was added. The reaction mixture was transferred to a centrifuge tube; the volume was filled up to $15 \mathrm{~mL}$ with hexane and the mixture was centrifuged at $5000 \mathrm{rpm}(2599 \mathrm{~g})$ for 10 $\min$. The supernatant of this centrifugation was diluted with 15 $\mathrm{mL}$ of methyl acetate to induce agglomeration of the NPLs and centrifuged again at $8000 \mathrm{rpm}(6654 \mathrm{~g})$ for $10 \mathrm{~min}$. The precipitate of this centrifugation was redispersed in $5 \mathrm{~mL}$ of hexane. The resulting solution was clear yellow and contained pure 3 ML NPLs.

Preparation of 0.1 M Se-ODE Solution. Briefly, $46.5 \mathrm{~mL}$ of ODE was introduced into a three-necked flask and degassed under vacuum for $15 \mathrm{~min}$ at $100{ }^{\circ} \mathrm{C}$. Afterward, ODE was heated to $180{ }^{\circ} \mathrm{C}$ under argon and $393 \mathrm{mg}$ (4.976 mmol) of selenium dispersed in $3.3 \mathrm{~mL}$ of toluene was added. The SeODE mixture was heated to $205{ }^{\circ} \mathrm{C}$ for $25 \mathrm{~min}$. During this period, the color of the mixture changed from gray to yellow. Then, the mixture was kept at $205{ }^{\circ} \mathrm{C}$ for $30 \mathrm{~min}$. Next, the solution was cooled to room temperature and transferred to a nitrogen-filled glovebox.

Seeded Growth of $3 \mathrm{ML}$ NPLs to Obtain Larger Lateral Sizes (NPL 600, NPL 710). We synthesized NPL 600 and NPL 710 from lateral extension of NPL 150. Briefly, $5 \mathrm{~mL}$ of ODE, $2 \mathrm{~mL}$ of NPL $150\left(8.40 \times 10^{-7} \mathrm{M}\right.$, based on absorption), $12.8 \mathrm{mg}$ of cadmium myristate, and $8.5 \mathrm{mg}$ of $\mathrm{Cd}(\mathrm{OAc})_{2} \cdot 2 \mathrm{H}_{2} \mathrm{O}$ were degassed at $100{ }^{\circ} \mathrm{C}$ for $15 \mathrm{~min}$. Then, the solution was heated to $190{ }^{\circ} \mathrm{C}$ under argon. Next, 1.0/1.5 $\mathrm{mL}$ of Se-ODE (for NPL 600/NPL 710, respectively) was introduced using a syringe pump at a speed of $50 \mu \mathrm{L} / \mathrm{min}$. The reaction was stopped by cooling to room temperature with a water bath. As the reaction was cooling, the syringe pump was stopped at $100{ }^{\circ} \mathrm{C}$ and $1 \mathrm{~mL}$ of oleic acid was added at $60{ }^{\circ} \mathrm{C}$. After reaching room temperature, the reaction mixture was diluted with $5 \mathrm{~mL}$ of methyl acetate to induce agglomeration of the NPLs and centrifuged at $8000 \mathrm{rpm}$ for $10 \mathrm{~min}$. The precipitate was redispersed with $5 \mathrm{~mL}$ of hexane. No side products from the formation of new crystal nuclei or sample losses from loss of NPLs were found after purification.

Synthesis of 4,7-Di(thiophen-2-yl)benzo[c][1,2,5]thiadiazole (DBT). ${ }^{27}$ 4,7-Dibromobenzo[c][1,2,5] thiadiazole $(1.15 \mathrm{~g}, 3.91 \mathrm{mmol})$ and tributyl(thiophen-2-yl)stannane $(2.73$ $\mathrm{mL}, 8.61 \mathrm{mmol}$ ) were added to a previously dried Schlenk tube and dissolved in dry THF $(40 \mathrm{~mL})$. The solution was degassed with argon for $15 \mathrm{~min} ; \mathrm{PdCl}_{2}\left(\mathrm{PPh}_{3}\right)_{2}(55 \mathrm{mg}, 0.08 \mathrm{mmol})$ was added, and the mixture was heated to $70{ }^{\circ} \mathrm{C}$ for $12 \mathrm{~h}$. After 
cooling to room temperature, THF was evaporated and the residue was taken up in DCM $(50 \mathrm{~mL})$. The organic phase was extracted with water $(3 \times 25 \mathrm{~mL})$ and brine $(25 \mathrm{~mL})$. After drying with anhydrous $\mathrm{MgSO}_{4}$ and filtration, the resulting solution was concentrated by evaporation. The crude product was recrystallized in methanol, followed by washing with cold hexanes (100 mL). 4,7-Di(thiophen-2-yl)benzo-2,1,3-thiadiazole was obtained as red needles $(1.07 \mathrm{~g}, 91 \%)$.

${ }^{1} \mathrm{H}$ NMR $\left(300 \mathrm{MHz}^{\mathrm{CDCl}}{ }_{3}\right) \delta 8.10(\mathrm{~d}, 2 \mathrm{H}), 7.83(\mathrm{~s}, 2 \mathrm{H})$, $7.45(\mathrm{~d}, 2 \mathrm{H}), 7.21(\mathrm{t}, 2 \mathrm{H}) \mathrm{ppm}$.

${ }^{13} \mathrm{C} \mathrm{NMR}\left(75 \mathrm{MHz}, \mathrm{CDCl}_{3}\right) \delta 152.72,139.47,128.13$, $127.62,126.92,126.07,125.86 \mathrm{ppm}$.

Synthesis of 5-(7-(Thiophen-2-yl)benzo[c][1,2,5]thiadiazol-4-yl)thiophene-2-carboxylic Acid (DBTCA). Step 1: In a dried Schlenk tube, DBT $(650 \mathrm{mg}, 2.16 \mathrm{mmol})$ and DMF (469 $\mu \mathrm{L}, 6.06 \mathrm{mmol})$ were dissolved in 1,2dichloroethane $(20 \mathrm{~mL}) . \mathrm{POCl}_{3}(217 \mu \mathrm{L}, 2.38 \mathrm{mmol})$ was slowly added to the solution. The mixture was stirred at $80{ }^{\circ} \mathrm{C}$ for $24 \mathrm{~h}$. After cooling to room temperature, a saturated ammonium acetate solution $(20 \mathrm{~mL})$ was added and was left under stirring for $30 \mathrm{~min}$. DCM $(20 \mathrm{~mL})$ was added, and the organic phase was extracted with water $(3 \times 20 \mathrm{~mL})$ and brine $(20 \mathrm{~mL})$. The combined organic phases were dried over $\mathrm{MgSO}_{4}$ and concentrated through rotary evaporation. The crude product was purified by column chromatography with hexanes/ethyl acetate (gradient from 2:1 to $0: 1 \mathrm{v} / \mathrm{v}$ ), and 5-(7(thiophen-2-yl)benzo[ $c][1,2,5]$ thiadiazol-4-yl)thiophene-2carbaldehyde $(380 \mathrm{mg}, 53 \%)$ was obtained as a red powder.

${ }^{1} \mathrm{H} \mathrm{NMR}\left(300 \mathrm{MHz}, \mathrm{CDCl}_{3}\right) \delta 9.97(\mathrm{~s}, 1 \mathrm{H}), 8.17(\mathrm{~s}, 2 \mathrm{H})$, $7.97(\mathrm{~d}, 1 \mathrm{H}), 7.89(\mathrm{~d}, 1 \mathrm{H}), 7.83(\mathrm{~d}, 1 \mathrm{H}), 7.50(\mathrm{~d}, 1 \mathrm{H}), 7.23(\mathrm{t}$, $1 \mathrm{H}) \mathrm{ppm}$.

${ }^{13} \mathrm{C}$ NMR (75 MHz, $\left.\mathrm{CDCl}_{3}\right) \delta 183.14,152.58,152.52$, $148.71,143.52,139.01,136.93,128.50,128.35,128.18,128.06$, 127.91, 127.51, 125.39, $124.46 \mathrm{ppm}$.

Step 2: In a dried Schlenk tube, 5-(7-(thiophen-2-yl)benzo$[c][1,2,5]$ thiadiazol-4-yl)thiophene-2-carbaldehyde $(150 \mathrm{mg}$, $0.46 \mathrm{mmol})$ was dissolved in acetone $(12 \mathrm{~mL})$ and the solution was cooled to $0{ }^{\circ} \mathrm{C}$ in an ice bath. Potassium permanganate $(87 \mathrm{mg}, 0.55 \mathrm{mmol})$ was added and the reaction was stirred at room temperature for $8 \mathrm{~h}$. The mixture was concentrated via rotary evaporation, and the residue was dissolved in a solution of $\mathrm{N}, \mathrm{N}$-diisopropylethylamine in acetonitrile (10 vol \%) and was left under stirring for $2 \mathrm{~h}$. A slurry of ion exchange resin (Dowex IX8 chloride form 200400 mesh) in acetonitrile was prepared and loaded into a short column. The product solution was then absorbed into the column and washed several times with acetonitrile and DCM. For elution, trifluoroacetic acid in DCM ( 5 vol \%) was passed through the column. After washing the resulting organic phase with brine and drying over $\mathrm{MgSO}_{4}$, the solution was concentrated to dryness. 5-(7-(Thiophen-2-yl)benzo[c]$[1,2,5]$ thiadiazol-4-yl)thiophene-2-carboxylic acid was obtained as a red powder (139 $\mathrm{mg}, 89 \%)$.

${ }^{1} \mathrm{H}$ NMR (500 MHz, DMSO- $\left.d_{6}, 373 \mathrm{~K}\right) \delta 8.14(\mathrm{~m}, 2 \mathrm{H})$, $8.05(\mathrm{~d}, 1 \mathrm{H}), 8.04(\mathrm{~d}, 1 \mathrm{H}), 7.79(\mathrm{~d}, 1 \mathrm{H}), 7.71(\mathrm{~d}, 1 \mathrm{H}), 7.25(\mathrm{t}$, $1 \mathrm{H}) \mathrm{ppm}$.

${ }^{13} \mathrm{C}$ NMR (125 MHz, DMSO- $\left.d_{6}, 373 \mathrm{~K}\right) \delta 162.27,151.50$, $151.49,144.04,137.98,135.12,132.87,127.97,127.64,127.58$, 127.12, 126.78, 126.28, 125.37, 123.87 ppm.

Sample Preparation for FRET Experiments. A stock solution of DBTCA was prepared by dissolving $1.6 \mathrm{mg}$ of pure powder in $33.78 \mathrm{~mL}$ of toluene $\left([\mathrm{DBTCA}]=1.375 \times 10^{-4}\right.$ M). Dispersions of NPL 150, NPL 600, and NPL 710 were diluted with $600 \mu \mathrm{L}$ of toluene in a $700 \mu \mathrm{L}$ quartz cuvette and adjusted to the same molar concentration of $4.87 \times 10^{-8} \mathrm{M}$. The NPL samples were characterized using absorption, steadystate, and time-correlated single-photon counting (TCSPC) PL spectroscopies. Then, $1 \mu \mathrm{L}$ of the DBTCA stock solution was mixed with the NPL dispersion and the samples were characterized again by absorption, steady-state, and TCSPC PL spectroscopies. This process was repeated until a ratio of $\sim 700$ DBTCA/NPL was reached.

Sample Preparation for Kinetic Experiments. NPL 150, NPL 600, and NPL 710 were diluted to a volume of 600 $\mu \mathrm{L}$ with toluene in a four-sided cuvette to molar concentrations of $1.95 \times 10^{-7}, 4.83 \times 10^{-8}$, and $4.0 \times 10^{-8} \mathrm{M}$, respectively, to reach the same absorption. Then, $40 \mu \mathrm{L}$ of the DBTCA stock solution (described in the previous paragraph) was added. The PL spectra were taken every second for a duration of $10 \mathrm{~min}$.

Transmission Electron Microscopy (TEM). To provide the TEM images of the NPLs, $10 \mu \mathrm{L}$ of a sample was diluted with hexane and drop-casted to a carbon-coated copper TEMgrid. Within $1 \mathrm{~min}$, the hexane fully evaporated. Afterward, the sample was analyzed with a JEOL JEM-1400 TEM at an acceleration voltage of $120 \mathrm{kV}$. The sizes of the NPLs were measured from the TEM images using ImageJ. The geometry of the NPLs was assumed to be perfect squares. For typical size determination, 100-200 NPLs were measured.

Steady-State Absorption Spectroscopy. The absorption spectra were measured using an Agilent Cary 60 spectrophotometer or Avantes spectrophotometer consisting of Avantes AvaLight-DH-S-BAL as the UV-vis light source passing through a neutral density filter (optical density $=2.0$ ) and fiber-coupled to an Avantes SensLine AvaSpec-HSC-TEC detector.

Steady-State Photoluminescence (PL) Spectroscopy. The photoluminescence spectra were recorded using an Avantes SensLine AvaSpec-HSC-TEC spectrophotometer in $90^{\circ}$ geometry. A Prizmatix Silver high-power LED was used as the excitation source (emission peak $369 \mathrm{~nm}$, FWHM $\sim 10$ $\mathrm{nm})$.

Time-Resolved PL Spectroscopy/Time-Correlated Single-Photon Counting (TCSPC). PL lifetime measurements were conducted with a FluoTime200 time-correlated single-photon counting setup. Samples were excited with a laser at $380 \mathrm{~nm}$, which was controlled by PicoQuant PDL 800D. The signal was detected using a microchannel plate photomultiplier tube that was connected to a PicoHarp 300 time-correlated single-photon counting system. NPL PL signals were read at $462 \mathrm{~nm}$, while DBTCA signals were read at $565 \mathrm{~nm}$. The instrument response function was measured using a dispersion of silica nanoparticles (LUDOX HS-40 colloidal silica) in water. Lifetimes of the NPLs were fitted with a double exponential function, while the lifetime of DBTCA was fitted with a monoexponential function.

Determination of Quantum Yields (QYs). Quantum yields were determined relative to a reference dye $^{34}$ and conducted in open air. Rhodamine $6 \mathrm{G}$ was dissolved in absolute ethanol. $3 \mathrm{ML}$ CdSe NPLs were diluted in hexane until the sample absorption approached a value of 0.1 at a wavelength of $370 \mathrm{~nm}$. The photoluminescence of the samples was recorded at this concentration using blue LED excitation at $369 \mathrm{~nm}$ (FWHM $10 \mathrm{~nm})$. Further dilution of the samples allowed us to obtain a linear fit between the sample absorption and the integrated photoluminescence, which was compared to 

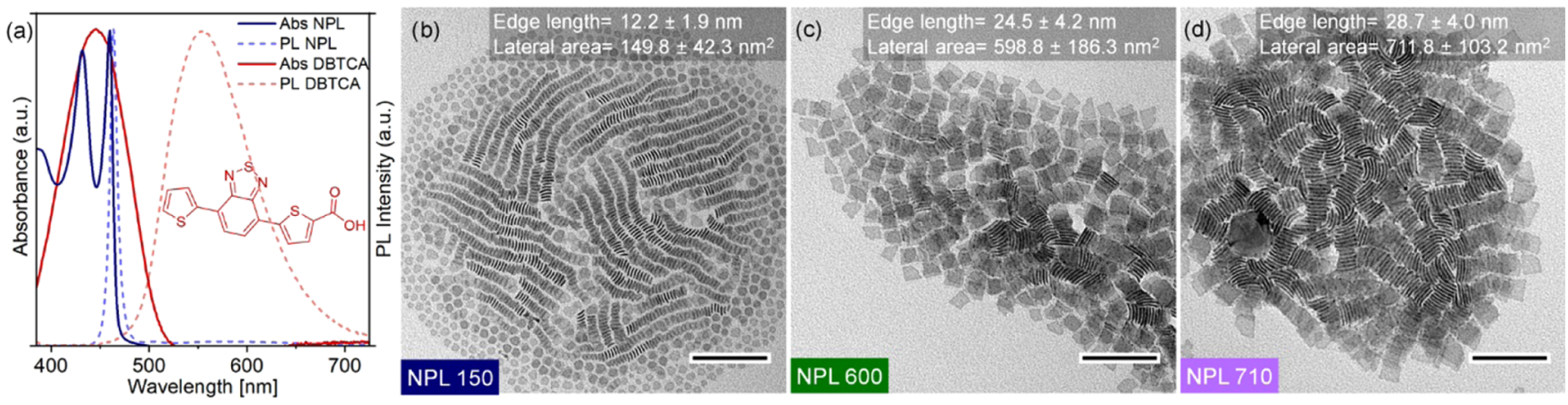

Figure 1. Properties of 3 ML CdSe NPLs and DBTCA. (a) Absorption (dark blue/red line) and PL spectra (pale blue/red dotted line) of NPLs/ DBTCA in toluene. The inset shows the structure of DBTCA. The overlap integral $J$ between the NPLs' PL emission and DBTCA's absorption is $2.2 \times 10^{15} \mathrm{~nm}^{4} \mathrm{M}^{-1} \mathrm{~cm}^{-1}$. (b) TEM image of the small NPLs with an average lateral area of $150 \mathrm{~nm}^{2}$. (c) TEM image of the medium NPLs with an average lateral area of $600 \mathrm{~nm}^{2}$. (d) TEM image of the large NPLs with an average lateral area of $710 \mathrm{~nm}^{2}$. Scale bars correspond to a length of 100 nm.

the Rhodamine $6 \mathrm{G}$ standard linear fit to obtain the quantum yield of the samples.

Isothermal Titration Calorimetry (ITC). ITC experiments were performed with a Nano ITC Low Volume from TA Instruments (Eschborn, Germany). The temperature was set to $25^{\circ} \mathrm{C}$ during all measurements. The effective cell volume was $170 \mu \mathrm{L}$, and a stirring rate of $350 \mathrm{rpm}$ was chosen for all experiments. During each experiment, $50 \mu \mathrm{L}$ of DBTCA $\left([\mathrm{DBTCA}]=1.0 \times 10^{-4} \mathrm{M}\right.$ in toluene $)$ was titrated into a toluene dispersion of NPLs with a lateral size of $454 \mathrm{~nm}^{2}$ (NPL $\left.450,[\mathrm{NPL} 450]=3.3 \times 10^{-8} \mathrm{M}\right)$ and titration steps of $25 \times 2$ $\mu \mathrm{L}$. Additionally, the same DBTCA solution was titrated into pure toluene to determine the heat of dilution. The temporal spacing between injections was set to $300 \mathrm{~s}$. The integrated heats for each titration were analyzed with an independent binding model after subtraction of the heat of dilution using the NanoAnalyze software, version 3.5.0, by TA Instruments. ${ }^{35}$ Measurements were performed in triplicate and fit parameter mean values and standard deviation were calculated from these measurements.

\section{RESULTS AND DISCUSSION}

Synthesis and Functionalization of 3 ML NPLs. To get a good spectral overlap between the NPLs and DBTCA, $3 \mathrm{ML}$ thick CdSe NPLs were selected as the donor (Figure 1a). The emission peak of $3 \mathrm{ML}$ NPLs lies at $462 \mathrm{~nm}$, close to the absorption maximum of DBTCA at $446 \mathrm{~nm}$. This results in a large overlap integral $J$ of $2.2 \times 10^{15} \mathrm{~nm}^{4} \mathrm{M}^{-1} \mathrm{~cm}^{-1}$.

To study the influence of the lateral size in FRET with DBTCA we first had to develop a synthesis pathway for highquality 3 ML NPLs of adjustable lateral dimensions. First, we synthesized laterally small NPLs (surface area of $\sim 150 \mathrm{~nm}^{2}$, Figure $1 \mathrm{~b}$ "NPL 150") with a modified protocol according to the work of Riedinger et al. ${ }^{33}$ These NPLs were laterally extended with a seeded growth synthesis based on the method developed by Tessier et al. ${ }^{36}$ to obtain a series of laterally larger NPLs (Figure 1c,d, "NPL 600" and "NPL 710", respectively).

Using our modified procedure, we were able to produce three differently sized NPLs with narrow lateral size distribution and a relatively regular shape (Figure $1 \mathrm{~b}, \mathrm{c}$ ). As expected, the lateral size extension increased the absorption of the NPLs linearly with the lateral area, which agrees with previous findings in $4 \mathrm{ML}$ and $5 \mathrm{ML}$ NPLs [see the Supporting Information (SI), Figure S1].
After having a series of differently sized 3 ML NPLs at hand, we first had to look into the chemistry of DBTCA with the NPL surface. Since FRET is strongly distance-dependent, it is important to know whether DBTCA, when mixed with NPLs in toluene, freely diffuses or is directly bound to the surface of the NPLs.

We analyzed this using isothermal titration calorimetry (ITC). If the binding of DBTCA to the surface atoms is favored by equilibrium, addition of DBTCA to the NPLs results in a heat release or absorption upon interaction of the carboxylic group with the Cd-terminated NPL surfaces. Note that the lack of a carboxylic group results in no binding (Figure S3). If the equilibrium disfavors binding, no appreciable heat change occurs due to a few products. In our experiments, the binding of DBTCA to NPLs' surface indeed absorbs heat (Figures 2 and S2), which shows that binding is favored and could also be used to quantify the maximum number of

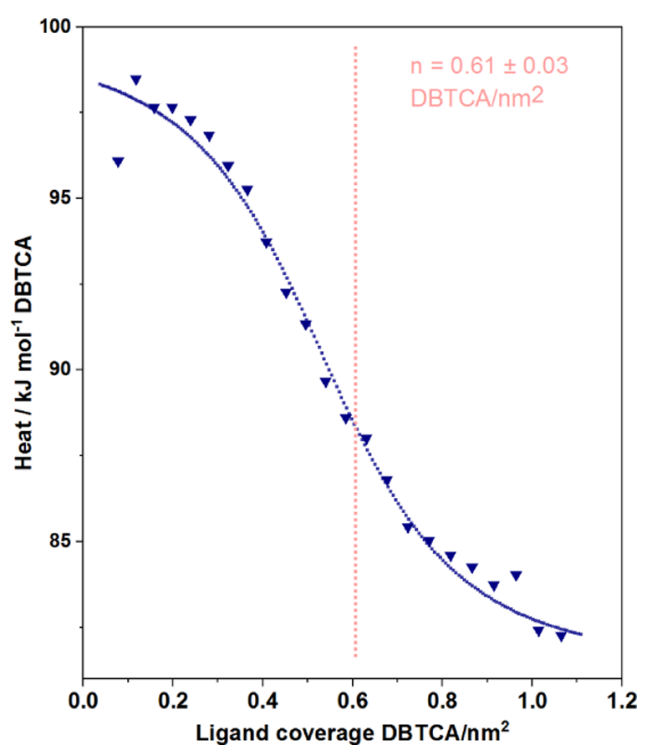

Figure 2. Exemplary ITC measurement of DBTCA titrated to NPL 450 in toluene. Results are shown in DBTCA coverage per $\mathrm{nm}^{2}$. Data shows integrated heats after subtraction of dilution heats together with an independent binding model fit. The molar ratio at full coverage corresponds to $0.61 \mathrm{DBTCA} / \mathrm{nm}^{2}$. For corrected heat rates of the titration of DBTCA to NPLs and into pure toluene (dilution experiment), see Figure S2. 
DBTCA per NPL. Based on the observed heat changes (negative Gibbs free energy, indicating surface binding), we determined the thermodynamic reaction parameters of the titration by careful fitting. We found that $\Delta H=20.4 \pm 1.2 \mathrm{~kJ} /$ $\mathrm{mol}, \Delta S=180.0 \pm 2.9 \mathrm{~J} / \mathrm{mol} \mathrm{K}, \Delta G=-33.2 \pm 0.4 \mathrm{~kJ} / \mathrm{mol}$, and $K_{\mathrm{d}}=1.6 \pm 0.2 \times 10^{-6} \mathrm{M}$. The negative Gibbs free energy in relation to positive enthalpy and entropy indicates that the process is entropically driven, most likely due to unlocking of the rotational degrees of freedom of the myristic acid released into solution after an exchange with DBTCA, further changes of the solvation shell, and inter-DBTCA $\pi-\pi$ interactions in solution. $^{37,38}$ Meanwhile, the small dissociation constant $K_{\mathrm{d}}$ indicates that the DBTCA binding to the NPLs is highly favored. Noteworthily, the entire NPL surface can participate in binding. This enhances the binding probability significantly, similar to the situation of macromolecules with multiple binding sites (for details, see Figure S4). ${ }^{39}$ We confirmed the favorable binding by kinetic experiments (Figure S5). This suggests that almost all DBTCA introduced to the system bind to the NPLs until the surface is fully covered. Furthermore, we observed the inflection point (reaction stoichiometry) at a ligand coverage of $0.61 \mathrm{DBTCA} / \mathrm{nm}^{2}$ (Figure 2). In terms of DBTCA/NPL, this corresponds to $1.8 \times 10^{2}, 7.0 \times 10^{2}$, and $8.4 \times 10^{2}$ DBTCA/NPL for NPLs 150, 600, and 710, respectively. When the DBTCA/NPL ratio exceeds these values, we should only see changes resulting from free excess DBTCA in solution, in the steady-state PL and time-resolved PL measurements of the NPLs and the DBTCA.

Estimation of Förster Radius Based on FRET Equation. The energy transfer from photoexcited semiconductor nanocrystals to suitable organic ligands can often be described through FRET-based mechanisms ${ }^{13,40-42}$ with few exceptions. ${ }^{17,43}$ For NPLs, this involves the interaction between the transition dipole of the exciton and the transition dipole of the fluorophore. When the NPLs are excited, the position of the exciton on the NPL is unknown. ${ }^{44}$ This means that we cannot use the traditional assumption of center-center distance of donor and acceptor since the donor (the exciton) exhibits a stochastic distribution of residence within the NPL. Thus, the distance between the donor and the acceptor must be measured from the center-to-center distance of the exciton and DBTCA rather than the center of NPLs (Scheme 1). However, since the position of the generated exciton is unknown, we must estimate this by statistical means using the DBTCA surface coverage. As the surface coverage increases, the likelihood of the exciton to be near DBTCA increases.

Energy transfer via FRET can occur efficiently as long as DBTCA lies within the Förster radius $\left(R_{0}\right)$ of NPL-DBTCA

Scheme 1. Surface Binding of DBTCA on the (100) Basal Planes of 3 ML CdSe NPLs ${ }^{a}$

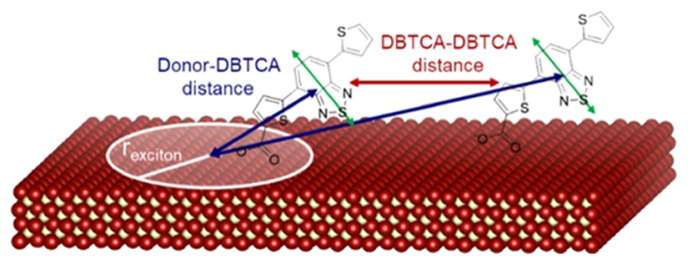

${ }^{a}$ The left-hand side DBTCA is positioned within the exciton radius, while the right is positioned outside the radius. Red atoms represent $\mathrm{Cd}$, while yellow atoms represent Se. Atomic sizes are not to scale. Green arrows indicate the dipole moment of DBTCA. and the orientation factor $\kappa^{2}$ for the conjugate is nonzero. Once the energy is transferred to DBTCA, the molecule can relax back to its ground state by emission of a photon. However, this process could be complicated by the possibility of (partial) self-quenching from dimerization/oligomerization of nearby DBTCA molecules ${ }^{45}$ on the surface of NPLs at high ligand coverage (refer to Figures S13 and S14).

Based on the properties of the three differently sized NPLs and DBTCA, $R_{0}$ can be estimated using eq $1^{46}$ to estimate expected distances for efficient FRET (Table 1).

$$
R_{0}^{6}=8.79 \times 10^{-11}\left(\mathrm{~nm}^{2} \mathrm{Mcm}\right) \times \frac{\Phi J \kappa^{2}}{n^{4}}
$$

Here, $\Phi$ is the quantum yield of the donor, $\kappa^{2}$ is the orientation factor between the donor and the acceptor, $n$ is the refractive index of the solvent, and $J$ is the overlap integral, given by eq $2^{47}$

$$
J=\int f_{\text {donor }}(\lambda) \varepsilon_{\text {acceptor }}(\lambda) \lambda^{4} \mathrm{~d} \lambda
$$

Here, $f$ is the normalized fluorescence intensity of the donor and $\varepsilon$ is the molar extinction coefficient of the acceptor. Since the transition dipole moment in NPLs is mainly oriented inplane, ${ }^{48}$ the orientation factor $\kappa^{2}$ changes when DBTCA binds to the basal planes or the side facet. Erdem et al. demonstrated that $\kappa^{2}$ is $1 / 3$ when a pointlike donor dipole interacts with the basal plane of NPLs but is enhanced to $5 / 6$ when it interacts with the side facet of the NPLs. ${ }^{44}$ Since small NPLs have a greater contribution of side facet area compared to the basal plane area (Table S3), we accounted for this difference for the determination of the effective orientation factor $\kappa_{\text {eff }}^{2}$ (Table 1 ).

$R_{0}$ of the NPLs are calculated using eq 1 and are found to lie in a similar range (Table 1 ). The differences are mainly due to the different QYs of the NPL samples (Figure S6 and Table 1). Note that the distance dependence in this work is not $\left(1 / r^{4}\right)$, unlike in the study by Erdem et al., ${ }^{44}$ since the distance dependence is determined by the dimensionality of the acceptor (DBTCA in our case) as shown by Martinez et al. ${ }^{49}$

Interestingly, $R_{0}$ of the homo-FRET between DBTCA molecules is in the same range as the FRET process between NPLs and DBTCA, which implies that self-quenching is always a possible decay pathway for DBTCA when its surface-bound concentration is sufficiently high.

Lateral Size-Dependent FRET. To assess size and concentration dependencies in NPL-DBTCA FRET couples experimentally, we conjugated various amounts of DBTCA with the NPLs by mixing the two components at different ratios in toluene. As shown in Figure $3 a-c$, the PL intensities of the 3 ML NPLs decrease with increasing concentrations of DBTCA, while the DBTCA PL intensities increase first and decrease again after a certain critical surface coverage is reached. Furthermore, we observed subsequently decreasing NPL PL lifetimes upon binding of DBTCA (Figures S8, S9, and S10). We confirm the FRET-based nature of these phenomena by analyzing the quenching of the donor (NPL) PL and the concomitant enhancement of the acceptor (DBTCA) PL in Figure S11. As predicted from $R_{0}$ values of DBTCA-DBTCA homo-FRET (Table 1), self-quenching starts to occur at high DBTCA/NPL ratios where the average DBTCA-DBTCA distance becomes increasingly shorter.

As a result of self-quenching, the DBTCA PL intensity peaks at certain DBTCA/NPL ratios (Figure 3d, dotted arrows). Beyond this point, self-quenching starts to become the 
Table 1. Estimated $\kappa_{\text {eff }}^{2}$ and $R_{0}$ of the Possible Donor-Acceptor Pairs in This Study ${ }^{a}$

\begin{tabular}{|c|c|c|c|c|}
\hline & NPL 150 & NPL 600 & NPL 710 & DBTCA \\
\hline $\mathrm{QY}_{\text {donor }}$ & $0.48 \pm 0.048$ & $0.05 \pm 0.005$ & $0.02 \pm 0.002$ & $0.80 \pm 0.080$ \\
\hline$J_{\text {donor } \rightarrow \text { DBTCA }}\left(\mathrm{nm}^{4} \mathrm{M}^{-1} \mathrm{~cm}^{-1}\right)$ & $2.20 \times 10^{15}$ & $2.20 \times 10^{15}$ & $2.20 \times 10^{15}$ & $5.36 \times 10^{14}$ \\
\hline estimated $\kappa_{\text {eff }}^{2}$ & 0.40 & 0.37 & 0.37 & 0.67 \\
\hline$R_{0 \text { donor } \rightarrow \text { DBTCA }}(\mathrm{nm})$ & 4.4 & 3.0 & 2.6 & 4.1 \\
\hline
\end{tabular}

${ }^{a}$ Estimation details are shown in Table S3.
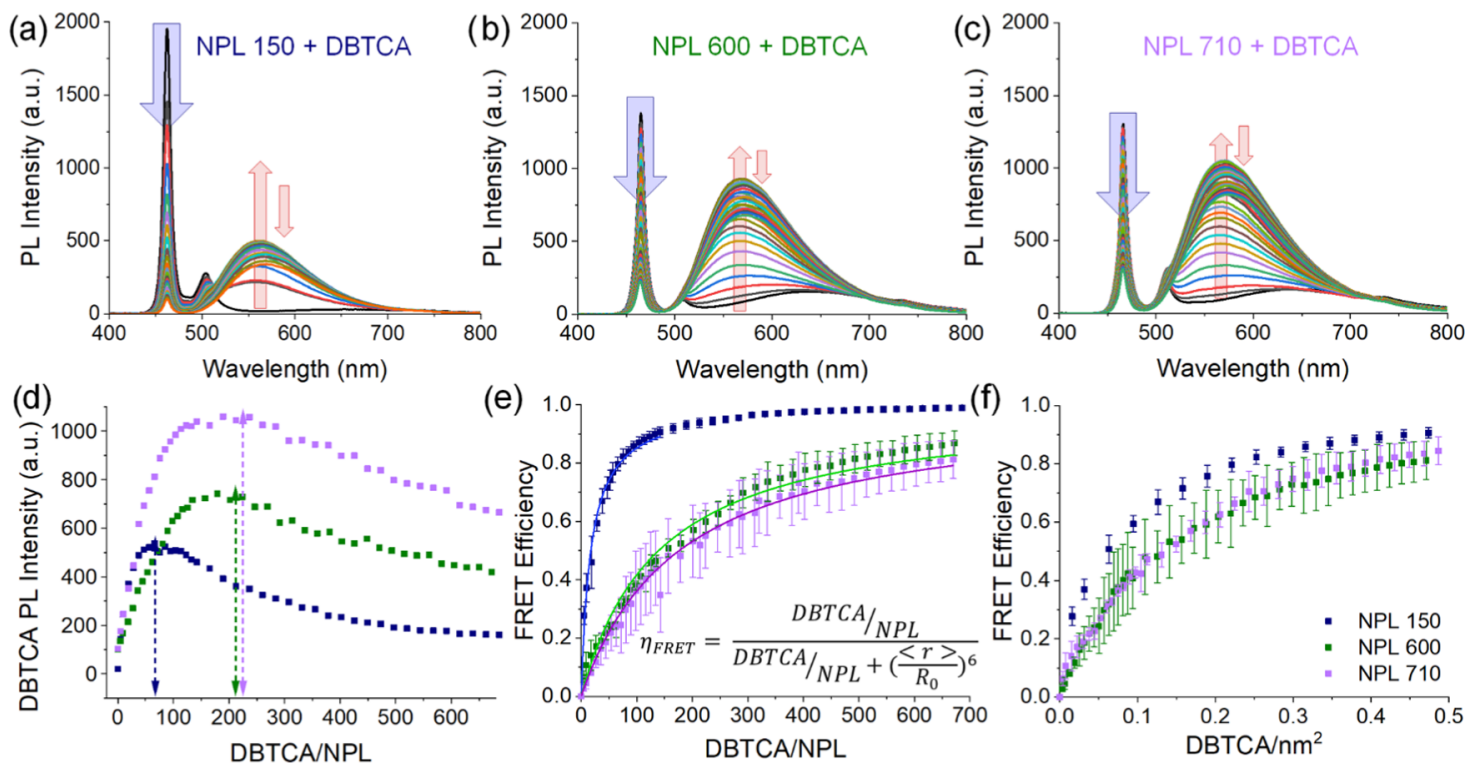

Figure 3. FRET between NPL and DBTCA at different DBTCA/NPL ratios. (a-c) PL spectra of differently sized NPLs with increasing DBTCA concentrations. Samples were excited with a blue LED $(\lambda=369 \mathrm{~nm}$, FWHM $\sim 10 \mathrm{~nm})$ where DBTCA weakly absorbs (Figure S7). NPL PL $(\lambda=$ $462 \mathrm{~nm})$ decreases as DBTCA concentration increases, while the DBTCA PL $\left(\lambda_{\max }=565 \mathrm{~nm}\right)$ increases. The small peak at $\sim 510 \mathrm{~nm}$ stems from a small amount of 4 ML CdSe NPL impurities. (d) Change of DBTCA steady-state PL intensity. PL increases from near zero at low DBTCA and reaches a maximum at a certain DBTCA/NPL ratio (dotted arrows) until the PL intensity decreases again. The position of the maximum PL shifts to higher DBTCA/NPL ratios for larger NPLs. (e) FRET efficiency calculated from donor quenching (eq 3) of differently sized NPLs at different DBTCA/NPL ratios. Solid lines indicate fits with eq 4. (f) FRET efficiency calculated from donor quenching of differently sized NPLs at equal DBTCA coverage.

Table 2. DBTCA/NPL Ratios and Ligand Coverage Values for the Three NPLs in Different Situations: Maximum DBTCA PL Intensity (from Figure 3d) and Maximum Coverage (Obtained from Figure 2, ITC Turnover Point) ${ }^{a}$

\begin{tabular}{|c|c|c|c|}
\hline & NPL 150 & NPL 600 & NPL 710 \\
\hline DBTCA/NPL at max. PL & $66 \pm 5$ & $216 \pm 13$ & $228 \pm 18$ \\
\hline DBTCA/NPL at max. coverage & $(1.8 \pm 0.1) \times 10^{2}$ & $(7.3 \pm 0.4) \times 10^{2}$ & $(8.7 \pm 0.5) \times 10^{2}$ \\
\hline DBTCA $/ \mathrm{nm}^{2}$ at max. PL & $0.22 \pm 0.02$ & $0.18 \pm 0.01$ & $0.16 \pm 0.01$ \\
\hline $\mathrm{DBTCA} / \mathrm{nm}^{2}$ at max. coverage & $0.61 \pm 0.03$ & $0.61 \pm 0.03$ & $0.61 \pm 0.03$ \\
\hline avg. DBTCA-DBTCA distance $(\mathrm{nm})$ at max. PL & 2.1 & 2.4 & 2.5 \\
\hline avg. DBTCA-DBTCA distance (nm) at max. coverage & 1.3 & 1.3 & 1.3 \\
\hline
\end{tabular}

dominant relaxation pathway. The maximum PL intensity is reached at 66, 216, and $228 \mathrm{DBTCA} / \mathrm{NPL}$ ratios for NPLs 150,600 , and 710 , respectively (Table 2 ).

The larger amount of DBTCA required to reach this maximum on larger NPLs can be more easily understood by looking at the PL normalized to the number of ligands per surface area of each NPL in the series (DBTCA $/ \mathrm{nm}^{2}$ ). As shown in Table 2 (third row), the coverage values for the three NPLs are in the same range, with smaller NPLs being more tolerant to higher coverage.

If we convert the coverage to the average separation distance of DBTCA-DBTCA (row 5), we obtain distances of 2.1-2.5 $\mathrm{nm}$. These distances are clearly below the calculated $R_{0}$ of
DBTCA-DBTCA (Table 1) and represents the point where self-quenching starts to dominate as the relaxation pathway.

The shorter DBTCA-DBTCA distance for small NPLs implies that there is some tolerance to having DBTCA close to one another before they efficiently quench. We attribute this to the higher relative amount of DBTCA ligands bound to the side facet that terminate the lateral expansion of the NPLs (Table S3). Binding to the small facets changes the orientation of the molecule and thus the orientation factors $\kappa^{2}$ for DBTCA homo-FRET and the desired NPL-DBTCA FRET. The orientation factor $\kappa^{2}$ of DBTCA-DBTCA decreases for cases where one molecule is bound to the narrow and one to the wide facets since they would be roughly orthogonally oriented 
to each other. Meanwhile, the NPL-DBTCA orientation factor increases, ${ }^{44}$ since the transition dipole moment of NPLs lie in their flat plane, ${ }^{50}$ opening the possibility of (nearly) coparallel transition dipole moment orientations in the best case. This difference, along with some unspecified losses observed in the large NPLs (Figure S11), could explain why NPL 710 need $\sim 3.5 \times$ more DBTCA to only reach $\sim 2 \times$ PL intensity compared to NPL 150.

The quenching of the NPL PL is calculated by eq 3 , where $\eta_{\text {FRET }}$ is the FRET efficiency. Moreover, in a first approximation, eq 4 has been fitted to the data keeping in mind that $r$ as well as $R_{0}$ can be widely distributed. ${ }^{47}$

$$
\begin{aligned}
& \eta_{\mathrm{FRET}}=1-\frac{F}{F_{0}} \\
& \eta_{\mathrm{FRET}}=\frac{\mathrm{DBTCA} / \mathrm{NPL}}{\mathrm{DBTCA} / \mathrm{NPL}+\left(\frac{<r>}{R_{0}}\right)^{6}}
\end{aligned}
$$

We made a plot of FRET efficiency as a function of DBTCA/ NPL ratios (Figure 3e) and fitted the data with eq 4 . These fits yielded $\langle r\rangle / R_{0}$ values of $1.62,2.28$, and 2.36 for NPL 150, 600 , and 710 , respectively. The lower $\langle r\rangle / R_{0}$ values for smaller NPLs indicate that it is easier to reach high FRET efficiencies using small NPLs at equal DBTCA/NPL ratios.

By looking at the DBTCA coverage (DBTCA $\left./ \mathrm{nm}^{2}\right)$ instead of DBTCA/NPL ratios, we found that the three NPLs exhibit similar FRET efficiencies at the same coverage (Figure 3f). This supports the idea that the excitons are localized rather than completely delocalized across the entire NPL, since the coverage shows the density of acceptors in the local proximity. Interestingly, when DBTCA $/ \mathrm{nm}^{2}>0.5$, the FRET efficiency obtained by donor quenching converges to a value of around 0.8-0.9. This happens close to when the surface of the NPLs is fully covered by DBTCA $\approx 0.61 \mathrm{DBTCA} / \mathrm{nm}^{2}$ (ITC, Figure 2 and Table 2). Based on this, we can conclude that the FRET efficiency between NPLs and DBTCA does reach its maximum at the highest possible ligand coverage.

However, if we compare the maximum DBTCA surface coverage to the surface coverage at maximum DBTCA PL (Table 2, rows 1 and 2), we find the maximum PL at a much lower surface coverage relative to the surface coverage where maximum FRET efficiency occurs. Therefore, we can conclude that although the energy is transferred to DBTCA efficiently when the surface is fully covered, it undergoes subsequent (partial) self-quenching processes. In other words, when using NPLs as sensitizers for fluorophores, it is best to minimize the self-quenching effects rather than maximize the FRET efficiency.

Qualitative Estimation of Ideal NPL Size for NPLDBTCA FRET. Since the excitons in NPLs have a small radius and their location of residence is stochastically distributed, the size of NPLs must play an important role. The higher the ratio of DBTCA/NPL, the denser the DBTCA packing on the NPL surface. At increasing ligand coverage, the probability of a DBTCA molecule to be situated near the exciton becomes higher. Therefore, if the FRET efficiencies are high at low DBTCA amounts, DBTCA must already be placed where it can interact with the exciton efficiently. We evaluated this tentatively by looking at the steepness of the slopes in Figure S12. The slope corresponds to the reciprocal probability of DBTCA being positioned next to the generated exciton. To interpret this parameter qualitatively, we fitted the curves with an exponential growth function (Figure S12a-c). The steepness of the exponential growth is measured by the denominator of the exponent and scales linearly with the lateral area of the NPLs (Figure 4a, gray line). While this fit is
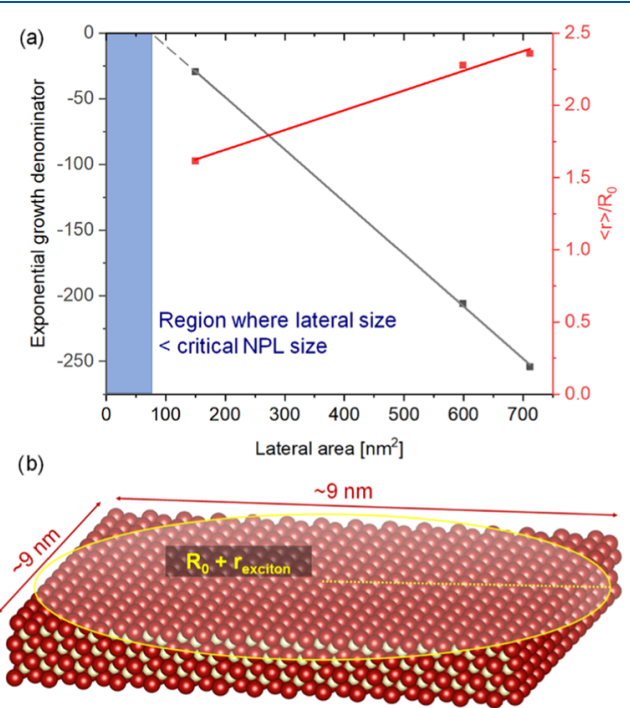

Figure 4. Determination of NPL size where DBTCA is always expected to be positioned close to the exciton. (a) Extrapolation of the exponential growth denominator determined from the fit of the slopes of Figure S12 using an exponential growth function. Results of the fits using eq 4 are shown in red for comparison. The fits do not include Poisson statistics since it introduces negligible changes of the results, smaller than the error margin of the measurements (Table S5). The denominator approaches zero at a lateral size of $78.5 \mathrm{~nm}^{2}$. (b) Sketch of square NPL with a lateral size $\approx 78.5 \mathrm{~nm}^{2}$, with an illustration of the exciton radius and $R_{0}$.

not based on a physical model, a linear dependency could also be obtained by fitting the curves using eq 4 (Figure $4 a$, red line; refer to Figure $3 \mathrm{e}$ for the corresponding fits). This finding agrees well with the assumed uniformly distributed density of excitons in NPLs by Erdem et al. ${ }^{44}$

When the exponential growth denominator approaches zero, the exponential growth function becomes infinitely steep. This means that the increase in FRET efficiency becomes infinitely fast as DBTCA binds to the NPLs' surface. In other words, DBTCA is always next to the exciton regardless of the binding site (for nonzero $\kappa_{\text {eff }}$ ). By extrapolation, we can obtain the critical NPL size where this phenomenon can occur (Figure 4a, blue region).

Since our study was carried out on roughly square NPLs, this size corresponds to square NPLs with dimensions of $\sim 9$ $\mathrm{nm} \times 9 \mathrm{~nm}$, slightly smaller than two times the sum of the exciton radius and average $R_{0, \text { avg }}$ (average overall $R_{0}$ in Table 1 $=3.3 \mathrm{~nm}$ ) for the NPL-DBTCA FRET couple (Figure $4 \mathrm{~b}$ ). Although the localization of the exciton and DBTCA binding is subject to statistical distributions, our qualitative estimation enables us to propose NPL sizes where high average FRET efficiencies could be reached without using high dye concentrations.

\section{CONCLUSIONS}

Our results on FRET between 3 ML CdSe NPLs and DBTCA ligands can be best described by dipole-dipole interactions between DBTCA and excitons in NPLs with radii in the range of a few nanometers, exhibiting stochastically distributed 
locations of residence in the NPL. We observed the influence of this distribution by comparing the FRET efficiency of NPL-DBTCA pairs, using differently sized NPLs. For laterally small NPLs, FRET efficiencies increase rapidly at low DBTCA/NPL ratios, which indicates that the probability of DBTCA to be positioned near the exciton is high. Conversely, for large NPLs, the FRET efficiency increases slowly until the conjugate reaches similar DBTCA surface densities, highlighting the dependence of FRET on surface coverage per unit area rather than DBTCA/NPL ratios. For smaller NPLs, there is a greater population of DBTCA that is positioned on the side facets, which experience less self-quenching compared to DBTCA positioned on the basal planes due to changes in the orientation factor. Therefore, increasing the absorption cross section of NPLs using large NPLs is accompanied by an increased separation between exciton and the acceptors, the most critical parameter in FRET $\left(1 / r^{6}\right)$. Thus, optimal sensitization by NPLs should aim at using smaller NPLs to enhance FRET from exciton to acceptor molecules. Noteworthily, even small NPLs have significantly higher absorption cross sections compared to their spherical counterparts ${ }^{51}$ that the coupled system could benefit from. From our findings and estimations, these small NPLs should be isotropic in the lateral dimensions (e.g., square), with the edge length smaller than 9 $\mathrm{nm}$ to maximize the probability of DBTCA being located close to the exciton. The fluorophore density should be around 0.2 fluorophores $/ \mathrm{nm}^{2}$ on the surface to minimize self-quenching. This result aids the design of efficient NPL-fluorophore systems for sensing, imaging, and photocatalysis.

\section{ASSOCIATED CONTENT}

\section{(s) Supporting Information}

The Supporting Information is available free of charge at https://pubs.acs.org/doi/10.1021/acs.jpcc.0c06199.

Absorption spectra of pure $3 \mathrm{ML}$ NPLs in hexane at an equal molar concentration; corrected heat rates of the titration of DBTCA to NPLs and into pure toluene; thermodynamic parameters from ITC; control experiment using DBT (DBTCA without the carboxylic acid group); ratio of bound DBTCA calculated from the dissociation constant from ITC; kinetics of DBTCA binding to 3 ML NPLs; lifetimes of the components obtained from exponential fitting; and DBTCA PL lifetime at increasing DBTCA/NPL ratios using NPL 150 with direct excitation to DBTCA (500 nm) (PDF)

\section{AUTHOR INFORMATION}

\section{Corresponding Author}

Andreas Riedinger - Max-Planck-Institute for Polymer

Research, 55128 Mainz, Germany; ○ orcid.org/0000-0002-

7732-0606; Email: riedinger@mpip-mainz.mpg.de

\section{Authors}

Henry Halim - Max-Planck-Institute for Polymer Research, 55128 Mainz, Germany

David Trieb - Max-Planck-Institute for Polymer Research, 55128 Mainz, Germany

Niklas Huber - Max-Planck-Institute for Polymer Research, 55128 Mainz, Germany

María Martínez-Negro - Max-Planck-Institute for Polymer Research, 55128 Mainz, Germany
Lars-Arne Meyer - Department of Chemistry, Johannes Gutenberg-University, 55099 Mainz, Germany

Thomas Basché - Department of Chemistry, Johannes Gutenberg-University, 55099 Mainz, Germany

Svenja Morsbach - Max-Planck-Institute for Polymer Research, 55128 Mainz, Germany; 이이.org/0000-0001-9662-8190

Kai A. I. Zhang - Max-Planck-Institute for Polymer Research, 55128 Mainz, Germany; @ orcid.org/0000-0003-0816-5718

Complete contact information is available at:

https://pubs.acs.org/10.1021/acs.jpcc.0c06199

\section{Author Contributions}

H.H. and D.T. contributed equally to this work. The manuscript was written through the contributions of all authors. All authors have given approval to the final version of the manuscript.

\section{Funding}

H.H. was supported by a fellowship from the Max Planck Graduate Center (MPGC), Mainz, Germany. N.H. was supported by the Kekule fellowship of Fonds der Chemischen Industrie (FCI) and the Gutenberg-Akademie of the Johannes Gutenberg University of Mainz.

\section{Notes}

The authors declare no competing financial interest.

\section{ACKNOWLEDGMENTS}

We thank Paul Kolpakov for the synthesis of cadmium myristate, Angelika Manhart for the synthesis of bis(stearoyl) selenide and Prof. Dr Katharina Landfester for the fruitful discussions.

\section{ABBREVIATIONS}

FRET, Förster resonance energy transfer; $R_{0}$, Förster radius; $\mathrm{PL}$, photoluminescence; NPLs, quasi-two dimensional semiconductor nanoplatelets; MLs, mono layers; DBT, 4,7-(2dithienyl)-2,1,3-benzothiadiazole; DBTCA, 5-(7-(thiophen-2yl)benzo[c] $[1,2,5]$ thiadiazol-4-yl)thiophene-2-carboxylic acid; QY, quantum yield; TEM, transmission electron microscopy; ITC, isothermal titration calorimetry; TCSPC, time-correlated single-photon counting

\section{REFERENCES}

(1) Lavis, L. D.; Raines, R. T. Bright Ideas for Chemical Biology. ACS Chem. Biol. 2008, 3, 142-155.

(2) Fan, J.; Hu, M.; Zhan, P.; Peng, X. Energy Transfer Cassettes Based on Organic Fluorophores: Construction and Applications in Ratiometric Sensing. Chem. Soc. Rev. 2013, 42, 29-43.

(3) Heilemann, M.; van de Linde, S.; Mukherjee, A.; Sauer, M. Super-Resolution Imaging with Small Organic Fluorophores. Angew. Chem., Int. Ed. 2009, 48, 6903-6908.

(4) Umezawa, K.; Citterio, D.; Suzuki, K. New Trends in nearInfrared Fluorophores for Bioimaging. Anal. Sci. 2014, 30, 327-349.

(5) Romero, N. A.; Nicewicz, D. A. Organic Photoredox Catalysis. Chem. Rev. 2016, 116, 10075-10166.

(6) Silvi, S.; Credi, A. Luminescent Sensors Based on Quantum Dot-Molecule Conjugates. Chem. Soc. Rev. 2015, 44, 4275-4289.

(7) Leatherdale, C. A.; Woo, W. K.; Mikulec, F. V.; Bawendi, M. G. On the Absorption Cross Section of Cdse Nanocrystal Quantum Dots. J. Phys. Chem. B 2002, 106, 7619-7622.

(8) Yeltik, A.; Delikanli, S.; Olutas, M.; Kelestemur, Y.; Guzelturk, B.; Demir, H. V. Experimental Determination of the Absorption Cross-Section and Molar Extinction Coefficient of Colloidal Cdse Nanoplatelets. J. Phys. Chem. C 2015, 119, 26768-26775. 
(9) Scott, R.; Achtstein, A. W.; Prudnikau, A.; Antanovich, A.; Christodoulou, S.; Moreels, I.; Artemyev, M.; Woggon, U. Two Photon Absorption in Ii-Vi Semiconductors: The Influence of Dimensionality and Size. Nano Lett. 2015, 15, 4985-4992.

(10) Bruchez, M.; Moronne, M.; Gin, P.; Weiss, S.; Alivisatos, A. P. Semiconductor Nanocrystals as Fluorescent Biological Labels. Science 1998, 281, 2013-2016.

(11) Schmelz, O.; Mews, A.; Basché, T.; Herrmann, A.; Müllen, K. Supramolecular Complexes from Cdse Nanocrystals and Organic Fluorophors. Langmuir 2001, 17, 2861-2865.

(12) Willard, D. M.; Carillo, L. L.; Jung, J.; Van Orden, A. Cdse-Zns Quantum Dots as Resonance Energy Transfer Donors in a Model Protein-Protein Binding Assay. Nano Lett. 2001, 1, 469-474.

(13) Clapp, A. R.; Medintz, I. L.; Mauro, J. M.; Fisher, B. R.; Bawendi, M. G.; Mattoussi, H. Fluorescence Resonance Energy Transfer between Quantum Dot Donors and Dye-Labeled Protein Acceptors. J. Am. Chem. Soc. 2004, 126, 301-310.

(14) Potapova, I.; Mruk, R.; Hübner, C.; Zentel, R.; Basché, T.; Mews, A. Cdse/Zns Nanocrystals with Dye-Functionalized Polymer Ligands Containing Many Anchor Groups. Angew. Chem., Int. Ed. 2005, 44, 2437-2440.

(15) Fernández-Argüelles, M. T.; Yakovlev, A.; Sperling, R. A.; Luccardini, C.; Gaillard, S.; Sanz Medel, A.; Mallet, J.-M.; Brochon, J.C.; Feltz, A.; Oheim, M.; et al. Synthesis and Characterization of Polymer-Coated Quantum Dots with Integrated Acceptor Dyes as Fret-Based Nanoprobes. Nano Lett. 2007, 7, 2613-2617.

(16) Zenkevich, E.; Cichos, F.; Shulga, A.; Petrov, E. P.; Blaudeck, T.; von Borczyskowski, C. Nanoassemblies Designed from Semiconductor Quantum Dots and Molecular Arrays. J. Phys. Chem. B 2005, 109, 8679-8692.

(17) Dayal, S.; Lou, Y.; Samia, A. C. S.; Berlin, J. C.; Kenney, M. E.; Burda, C. Observation of Non-Förster-Type Energy-Transfer Behavior in Quantum Dot-Phthalocyanine Conjugates. J. Am. Chem. Soc. 2006, 128, 13974-13975.

(18) Dworak, L.; Matylitsky, V. V.; Ren, T.; Basché, T.; Wachtveitl, J. Acceptor Concentration Dependence of Förster Resonance Energy Transfer Dynamics in Dye-Quantum Dot Complexes. J. Phys. Chem. C 2014, 118, 4396-4402.

(19) Clapp, A. R.; Medintz, I. L.; Mattoussi, H. Förster Resonance Energy Transfer Investigations Using Quantum-Dot Fluorophores. ChemPhysChem 2006, 7, 47-57.

(20) Ithurria, S.; Dubertret, B. Quasi 2d Colloidal Cdse Platelets with Thicknesses Controlled at the Atomic Level. J. Am. Chem. Soc. 2008, 130, 16504-16505.

(21) Ithurria, S.; Tessier, M. D.; Mahler, B.; Lobo, R. P. S. M.; Dubertret, B.; Efros, A. L. Colloidal Nanoplatelets with TwoDimensional Electronic Structure. Nat. Mater. 2011, 10, 936-941.

(22) Brumberg, A.; Harvey, S. M.; Philbin, J. P.; Diroll, B. T.; Lee, B.; Crooker, S. A.; Wasielewski, M. R.; Rabani, E.; Schaller, R. D. Determination of the in-Plane Exciton Radius in 2d Cdse Nanoplatelets Via Magneto-Optical Spectroscopy. ACS Nano 2019, 13, 8589-8596.

(23) Li, Q.; Liu, Q.; Schaller, R. D.; Lian, T. Reducing the Optical Gain Threshold in Two-Dimensional Cdse Nanoplatelets by the Giant Oscillator Strength Transition Effect. J. Phys. Chem. Lett. 2019, 10, 1624-1632.

(24) Morgan, D. P.; Kelley, D. F. Exciton Localization and Radiative Lifetimes in Cdse Nanoplatelets. J. Phys. Chem. C 2019, 123, 1866518675.

(25) Zhou, E.; Yamakawa, S.; Zhang, Y.; Tajima, K.; Yang, C.; Hashimoto, K. Indolo[3,2-B]Carbazole-Based Alternating DonorAcceptor Copolymers: Synthesis, Properties and Photovoltaic Application. J. Mater. Chem. 2009, 19, 7730-7737.

(26) Zhang, X.; Steckler, T. T.; Dasari, R. R.; Ohira, S.; Potscavage, W. J.; Tiwari, S. P.; Coppée, S.; Ellinger, S.; Barlow, S.; Brédas, J.-L.; et al. Dithienopyrrole-Based Donor-Acceptor Copolymers: Low Band-Gap Materials for Charge Transport, Photovoltaics and Electrochromism. J. Mater. Chem. 2010, 20, 123-134.
(27) Wang, L.; Huang, W.; Li, R.; Gehrig, D.; Blom, P. W. M.; Landfester, K.; Zhang, K. A. I. Structural Design Principle of SmallMolecule Organic Semiconductors for Metal-Free, Visible-LightPromoted Photocatalysis. Angew. Chem., Int. Ed. 2016, 55, 97839787.

(28) Wang, L.; Rörich, I.; Ramanan, C.; Blom, P. W. M.; Huang, W.; Li, R.; Zhang, K. A. I. Electron Donor-Free Photoredox Catalysis Via an Electron Transfer Cascade by Cooperative Organic Photocatalysts. Catal. Sci. Technol. 2018, 8, 3539-3547.

(29) Han, L.; Qin, D.; Jiang, X.; Liu, Y.; Wang, L.; Chen, J.; Cao, Y. Synthesis of High Quality Zinc-Blende Cdse Nanocrystals and Their Application in Hybrid Solar Cells. Nanotechnology 2006, 17, 47364742.

(30) Bertrand, G. H. V.; Polovitsyn, A.; Christodoulou, S.; Khan, A. H.; Moreels, I. Shape Control of Zincblende Cdse Nanoplatelets. Chem. Commun. 2016, 52, 11975-11978.

(31) Jiang, Y.; Ojo, W.-S.; Mahler, B.; Xu, X.; Abécassis, B.; Dubertret, B. Synthesis of Cdse Nanoplatelets without Short-Chain Ligands: Implication for Their Growth Mechanisms. ACS Omega 2018, 3, 6199-6205.

(32) Koketsu, M.; Nada, F.; Hiramatsu, S.; Ishihara, H. Reactions of Acyl Chlorides with Lialhseh. Preparation of Diacyl Selenides, Diacyl Diselenides, Selenocarboxylates and Cyclic Selenoanhydrides. J. Chem. Soc., Perkin Trans. 1 2002, 737-740.

(33) Riedinger, A.; Mule, A. S.; Knüsel, P. N.; Ott, F. D.; Rossinelli, A. A.; Norris, D. J. Identifying Reactive Organo-Selenium Precursors in the Synthesis of Cdse Nanoplatelets. Chem. Commun. 2018, 54, 11789-11792.

(34) Resch-Genger, U.; Rurack, K. Determination of the Photoluminescence Quantum Yield of Dilute Dye Solutions (Iupac Technical Report). Pure Appl. Chem. 2013, 85, 2005-2013.

(35) Müller, J.; Prozeller, D.; Ghazaryan, A.; Kokkinopoulou, M.; Mailänder, V.; Morsbach, S.; Landfester, K. Beyond the Protein Corona - Lipids Matter for Biological Response of Nanocarriers. Acta Biomater. 2018, 71, 420-431.

(36) Tessier, M. D.; Spinicelli, P.; Dupont, D.; Patriarche, G.; Ithurria, S.; Dubertret, B. Efficient Exciton Concentrators Built from Colloidal Core/Crown Cdse/Cds Semiconductor Nanoplatelets. Nano Lett. 2014, 14, 207-213.

(37) Turcu, I.; Mic, M. Size Dependence of Molecular SelfAssembling in Stacked Aggregates. 2. Heat Exchange Effects. J. Phys. Chem. B 2013, 117, 9083-9093.

(38) Miyamura, K.; Mihara, A.; Fujii, T.; Gohshi, Y.; Ishii, Y. Unusually Strong Interactions Mediated by Both.Pi.-.Pi. Stacking and Ch-Pi. Interactions Present in the Dimer of Nickel(Ii) Complex Coordinated with N-Butyl-Substituted Salen. J. Am. Chem. Soc. 1995, $117,2377-2378$.

(39) Hans, B. Enzyme Kinetics Principles and Methods; Wiley-VCH: Weinheim, 2008.

(40) Medintz, I. L.; Clapp, A. R.; Mattoussi, H.; Goldman, E. R.; Fisher, B.; Mauro, J. M. Self-Assembled Nanoscale Biosensors Based on Quantum Dot Fret Donors. Nat. Mater. 2003, 2, 630-638.

(41) Snee, P. T.; Tyrakowski, C. M.; Page, L. E.; Isovic, A.; Jawaid, A. M. Quantifying Quantum Dots through Förster Resonant Energy Transfer. J. Phys. Chem. C 2011, 115, 19578-19582.

(42) Ren, T.; Mandal, P. K.; Erker, W.; Liu, Z.; Avlasevich, Y.; Puhl, L.; Müllen, K.; Basché, T. A Simple and Versatile Route to Stable Quantum Dot-Dye Hybrids in Nonaqueous and Aqueous Solutions. J. Am. Chem. Soc. 2008, 130, 17242-17243.

(43) Kowerko, D.; Schuster, J.; Amecke, N.; Abdel-Mottaleb, M.; Dobrawa, R.; Würthner, F.; von Borczyskowski, C. Fret and Ligand Related Non-Fret Processes in Single Quantum Dot-Perylene Bisimide Assemblies. Phys. Chem. Chem. Phys. 2010, 12, 4112-4123.

(44) Erdem, O.; Gungor, K.; Guzelturk, B.; Tanriover, I.; Sak, M.; Olutas, M.; Dede, D.; Kelestemur, Y.; Demir, H. V. OrientationControlled Nonradiative Energy Transfer to Colloidal Nanoplatelets: Engineering Dipole Orientation Factor. Nano Lett. 2019, 19, 42974305. 
(45) Conroy, E. M.; Li, J. J.; Kim, H.; Algar, W. R. Self-Quenching, Dimerization, and Homo-Fret in Hetero-Fret Assemblies with Quantum Dot Donors and Multiple Dye Acceptors. J. Phys. Chem. C 2016, 120, 17817-17828.

(46) van der Meer, B. W.; Coker, G.; Chen, S. Y. S. Resonance Energy Transfer: Theory and Data; VCH: New York, 1994.

(47) Lakowicz, J. R. Principles of Fluorescence Spectroscopy; Springer: New York, 2007.

(48) Scott, R.; Heckmann, J.; Prudnikau, A. V.; Antanovich, A.; Mikhailov, A.; Owschimikow, N.; Artemyev, M.; Climente, J. I.;

Woggon, U.; Grosse, N. B.; et al. Directed Emission of Cdse Nanoplatelets Originating from Strongly Anisotropic 2d Electronic Structure. Nat. Nanotechnol. 2017, 12, 1155-1160.

(49) Hernández-Martínez, P. L.; Govorov, A. O.; Demir, H. V. Generalized Theory of Förster-Type Nonradiative Energy Transfer in Nanostructures with Mixed Dimensionality. J. Phys. Chem. C 2013, 117, 10203-10212.

(50) Gao, Y.; Weidman, M. C.; Tisdale, W. A. Cdse Nanoplatelet Films with Controlled Orientation of Their Transition Dipole Moment. Nano Lett. 2017, 17, 3837-3843.

(51) Achtstein, A. W.; Antanovich, A.; Prudnikau, A.; Scott, R.; Woggon, U.; Artemyev, M. Linear Absorption in Cdse Nanoplates: Thickness and Lateral Size Dependency of the Intrinsic Absorption. J. Phys. Chem. C 2015, 119, 20156-20161. 